\title{
artigo
}

Delfino, J.A.; Melo, A.K.P.; Bernardes, I.A.S.; Diniz, A.L.X.; Almeida, C.S.; Souza, D.A.S.;

Ações educativas para a gestante no pré-natal acerca dos cuidados com recém-nascido

\section{Ações educativas para a gestante no pré-natal acerca dos cuidados com recém-nascido}

\author{
Educational actions for pregnant women on newborn care \\ Acciones educativas para mujeres embarazadas no prenatal sobre el cuidado del recién nacido
}

\begin{abstract}
RESUMO
Objetivo: Identificar as ações educativas realizadas no pré-natal voltadas para os cuidados com o recém-nascido em Unidades Básicas de Saúde de um município do interior de Minas Gerais. Método: Estudo descritivo, de abordagem qualitativa que contou com a participação de dez puérperas cujo pré-natal foi realizado exclusivamente em Unidades Básicas de Saúde em um município do interior de Minas Gerais. A coleta de dados ocorreu por meio de entrevistas individuais e posteriormente, foi realizada a análise de conteúdo temático categorial. Resultados: Foram encontradas três categorias: 1) Início do pré-natal e qualidade das consultas; 2) Orientações recebidas durante as consultas do pré-natal, natureza destas informações e dúvidas das puérperas e 3) Educação em saúde em grupos de gestantes. Conclusão: As ações educativas para a gestante no pré-natal contemplam majoritariamente sobre a amamentação, aumentando as dificuldades das puérperas sobre outros cuidados com o recém-nascido. DESCRITORES: Educação em Saúde; Cuidado Pré-Natal; Recém-Nascido.
\end{abstract}

\section{ABSTRACT}

Objective: To identify the educational actions carried out in prenatal care aimed at the care of the newborn in Basic Health Units in a city in the interior of Minas Gerais. Method: Descriptive study, with a qualitative approach, with the participation of ten puerperal women whose prenatal care was performed exclusively in Basic Health Units in a city in the interior of Minas Gerais. Data collection took place through individual interviews and subsequently, thematic content analysis was carried out. Results: Three categories were found: 1) Start of prenatal care and quality of consultations; 2) Orientations received during prenatal consultations, nature of this information and doubts of the puerperal women and 3) Health education in groups of pregnant women. Conclusion: The educational actions for pregnant women in prenatal care mostly contemplate breastfeeding, increasing the difficulties of puerperal women about other care for the newborn.

DESCRIPTORS: Health Education; Prenatal Care; Newbor.

\section{RESUMEN}

Objetivo: Identificar las acciones educativas realizadas en atención prenatal dirigidas a la atención del recién nacido en Unidades Básicas de Salud de una ciudad del interior de Minas Gerais. Método: Estudio descriptivo, con abordaje cualitativo, con la participación de diez puérperas cuya atención prenatal se realizó exclusivamente en Unidades Básicas de Salud de una ciudad del interior de Minas Gerais. La recolección de datos se realizó a través de entrevistas individuales y posteriormente, se realizó un análisis de contenido temático. Resultados: Se encontraron tres categorías: 1) Inicio de la atención prenatal y calidad de las consultas; 2) Orientaciones recibidas durante las consultas prenatales, naturaleza de esta información y dudas de las puérperas y 3) Educación para la salud en grupos de gestantes. Conclusión: Las acciones educativas para gestantes en atención prenatal contemplan mayoritariamente la lactancia materna, aumentando las dificultades de las puérperas sobre otros cuidados del recién nacido.

DESCRIPTORES: Educación en Salud; Atención Prenatal; Posmaduro.

RECEBIDO EM: 15/12/2020 APROVADO EM: 11/01/2021

\section{Jaqueline Aparecida Delfino}

Graduada em Enfermagem. Universidade do Estado de Minas Gerais, Unidade Divinópolis.

ORCID: 0000-0002-5159-5724

\section{Anne Karen Pereira Melo}

Acadêmica de Enfermagem. Universidade do Estado de Minas Gerais, Unidade Divinópolis.

ORCID: 0000-0003-1212-2120 


\section{Isabelle Aparecida de Sousa Bernardes}

Acadêmica de Enfermagem. Universidade do Estado de Minas Gerais, Unidade Divinópolis. ORCID: 0000-0002-5147-2222

\section{Ana Luiza Xisto Diniz}

Acadêmica de Enfermagem. Universidade do Estado de Minas Gerais, Unidade Divinópolis. ORCID: 0000-0002-9872-7771

\section{Camila Souza de Almeida}

Enfermeira. Mestre em Ciências. Doutora pela Escola de Enfermagem da UFMG. Docente da Universidade do Estado de Minas Gerais, Unidade Divinópolis.

ORCID: 0000-0002-7032-0945

\section{Débora Aparecida Silva Souza}

Enfermeira. Mestre em Educação em Saúde e Enfermagem. Docente da Universidade do Estado de Minas Gerais, Unidade Divinópolis.

ORCID: 0000-0002-8937-584X

\section{INTRODUÇÃO}

A assistência ao pré-natal visa proporcionar um parto seguro, bem-estar neonatal e materno por meio da prevenção e/ou detecção precoce de doenças que possam interferir no desenvolvimento saudável do recém-nascido $(\mathrm{RN})$, além de oferecer riscos à saúde da gestante. ${ }^{1}$

Os profissionais da saúde, médicos e enfermeiros, são os responsáveis pelas consultas do pré-natal na Atenção Primária à Saúde. ${ }^{2}$ Possuem papel fundamental para atender às reais necessidades das gestantes e, sobretudo, prepará-la para a nova vida com o nascimento do recém-nascido. ${ }^{2-3}$

No entanto, além das ações sobre a saúde materno-infantil, é relevante orientar e promover às gestantes conhecimentos sobre os cuidados com o RN, ajudá-la a desenvolver habilidades para o cuidado após o nascimento no que se refere à higiene, amamentação, imunizações, sono, conforto do RN, cuidados com o coto umbilical, cuidados com o manejo da criança que ainda não sustenta a cabeça, posição correta para dormir, cuidados durante o banho, produtos que devem ser evitados na pele do recém-nascido, importância e necessidade das imunizações, dentre outros. ${ }^{1-4}$

Para que isso seja possível, uma alternativa é realizar a educação em saúde nas consultas de pré-natal. Entende-se por educação em saúde a promoção do conhecimento, favorecendo mudanças de com- portamentos que estimulem a autonomia e a capacidade para o cuidado de si conforme suas necessidades. ${ }^{5}$

No contexto da assistência ao pré-natal na Atenção Primária à Saúde, as ações educativas realizadas durante o pré-natal são importantes por permitir a construção de conhecimentos das gestantes acerca dos cuidados com o RN, visto que a realidade da puérpera é, na maioria das vezes, cercada de anseios e de fragilidade psíquicas, que pode interferir no cuidado efetivo do recém-nascido. ${ }^{6-7}$

Segundo estudo realizado no Brasil, a assistência ao pré-natal possui cobertura no país de $90 \%$, mas apenas $60 \%$ das gestantes foram orientadas sobre a amamentação no pré-natal e outros cuidados relacionados com o recém-nascido não foram citados. Este estudo concluiu sobre a baixa adequação da assistência no pré-natal. ${ }^{8}$ Corroborando com este resultado, uma pesquisa realizada com mulheres que realizaram o pré-natal na rede básica de saúde para avaliação da qualidade do pré-natal entre os anos 2013 e 2014, evidenciou que $98,9 \%$ das entrevistadas tinham realizado o pré-natal. Destas, $60 \%$ relataram que receberam orientações voltadas para a amamentação. ${ }^{9}$

Outros estudos mostram falhas nas ações educativas para as gestantes de baixo risco, observando que as gestantes aos nove meses de gestação ainda têm dúvidas em relação ao parto e a amamentação. ${ }^{2-10}$ Estes dados revelam que ainda são escassos os estudos que descrevem a realização da educação em saúde para os cuidados com o RN além da amamentação. De modo geral, a literatura disponível revela que no pré-natal prioriza-se orientações sobre a realização de exames laboratoriais e de imagem, cuidados referentes à alimentação da gestante, uso abusivo de substâncias alcoólicas e tóxicas e práticas de atividade física. ${ }^{2-11}$

Com relação ao período puerperal é enfatizada apenas a educação visando à amamentação e a pega correta, o que não permite que a puérpera torne-se protagonista no processo de cuidado com o RN, ficando muitas vezes vulnerável a cuidados inadequados que poderiam ter sido ensinados no pré-natal. ${ }^{2-11}$ Segundo uma pesquisa brasileira a ausência da propagação desses cuidados tem sido um desafio para reduzir a mortalidade infantil no país. ${ }^{4}$

A literatura também aponta que a atenção no puerpério não está consolidada nos serviços de saúde e, com isso, é comum a mulher retornar na unidade no primeiro mês de vida do recém-nascido desinformada sobre os cuidados com a criança. ${ }^{5}$

Desta maneira, este estudo foi guiado pela pergunta norteadora: quais as ações educativas sobre os cuidados com o recém-nascido são desenvolvidas durante o pré-natal? Para investigar este problema, o estudo traçou como objetivo identificar as ações educativas realizadas no pré-natal voltadas para os cuidados com o recém- 
-nascido nas Unidades Básicas de Saúde de um município do interior de Minas Gerais. Espera-se que os resultados possam somar com a literatura sobre a importância dos cuidados com o recém-nascido serem introduzidos ainda nas consultas de pré-natal.

\section{MÉTODOS}

Trata-se de estudo descritivo, de abordagem qualitativa. A pesquisa qualitativa pressupõe a investigação de fenômenos humanos com singularidades, permitindo a atribuição de significados aos fatos e às pessoas em interações sociais que podem ser descritas e analisadas qualitativamente. ${ }^{12} \mathrm{~A}$ metodologia adequa-se a este estudo, tendo em vista a análise do fenômeno "ações educativas no pré-natal sobre os cuidados com o recém-nascido", e a necessidade de imersão do pesquisador no campo para melhor conhecer como as gestantes são orientadas acerca dos cuidados com o recém-nascido.

$\mathrm{O}$ cenário do presente estudo foi o município de Divinópolis, região Centro-Oeste do estado de Minas Gerais, Brasil, que apresenta uma população de 240.408 habitantes, segundo último censo do ano de $2020 .{ }^{13}$

Os sujeitos entrevistados foram puérperas provenientes das Unidades Básicas de Saúde (UBS) que consiste no modelo de assistência à saúde, integrante da Atenção Primária à Saúde que se orienta pelos princípios do Sistema Único de Saúde. No município em estudo, foram atendidas 1.376 gestantes de janeiro de 2019 até setembro deste mesmo ano na rede pública de saúde. As UBS são compostas por uma equipe multiprofissional com médico generalista, médico ginecologista e obstetrícia, enfermeiro, técnico de enfermagem, dentista e psicólogo. ${ }^{14}$

As participantes do estudo foram dez puérperas que realizaram o pré-natal nas Unidades Básicas de Saúde. Os critérios de inclusão foram puérperas mães do primeiro filho e em acompanhamento exclusivo em Unidades Básicas de Saúde. Foram excluídas puérperas que não realizaram exclusivamente o pré-natal nas UBS e as que se negaram a participar.

Primeiramente, os pesquisadores realizariam a coleta dos dados nas UBS que realizam a vacina da $B C G$ em Divinópolis em janeiro de 2020. A escolha destas unidades se justificou para que se conseguisse alcançar o maior número de puérperas, pois estas mulheres estariam na unidade entre o 50 e 8 o dia de vida do recém-nascido para receber a vacina BCG. Entretanto, o início da coleta de dados coincidiu com a pandemia do novo Coronavírus (SAR$\mathrm{S}$-CoV-2), com isso as entrevistas foram realizadas via telefone conforme disponibilidade das participantes. Todas as entrevistas foram agendadas previamente mediante contato telefônico, para posteriormente a realização das entrevistas conforme a disponibilidade de cada puérpera.

Desta forma, o estudo foi apresentado para as participantes via contato telefônico e foram entregues nas residências o Termo de Consentimento Livre e Esclarecido (TCLE) que foi deixado na caixa de correios das residências. Após concordância, esclarecida as dúvidas das participantes via telefone e assinatura, as pesquisadoras realizaram a busca do TCLE. Ressalta-se que tanto para a entrega quanto para a busca do TCLE, foram atendidas as medidas sanitárias de segurança impostas pelo Coronavírus, como distanciamento social, uso de máscaras e higiene das mãos com álcool $70 \%$ em detrimento do Coronavírus.

Para a coleta de dados foram realizadas entrevistas a partir de um roteiro semiestruturado, elaborado de maneira conjunta pelas pesquisadoras conforme discussões vigentes na literatura sobre a temática. As participantes foram questionadas sobre: dados sociais, dados obstétricos e perguntas abertas referentes às consultas do pré-natal e seu conteúdo.

Por se tratar de uma pesquisa qualitativa não se delimitou o número de participantes, porque o objetivo não foi verificar a frequência, mas sim, explorar a variedade e a riqueza das informações. Desta forma, foi considerada a saturação de dados a partir do momento que as entrevistas não trouxerem novas informações ou novos dados. ${ }^{15}$
As entrevistas foram transcritas, sistematizadas e categorizados para compor um banco de dados. A técnica utilizada para a análise qualitativa dos dados baseou-se no método de análise de conteúdo temático-categorial proposto por Bardin. ${ }^{16}$ Após a transcrição das falas, foi realizada a etapa de análise textual composta pelas seguintes fases: 1) pré-análise, com leitura parcialmente orientada do material, para que o pesquisador pudesse se aproximar dos conteúdos expressos; 2) exploração do material, durante a qual o material foi organizado para que as idéias iniciais fossem sistematizadas e 3) tratamento dos resultados, processo em que todo o material foi separado em unidades de registro sobre cada tema e categoria.

A análise de conteúdo foi realizada com a finalidade de descrever e interpretar o conteúdo de toda classe de documentos e textos. O objetivo foi reinterpretar as mensagens e atingir uma compreensão sobre as ações educativas realizadas no pré-natal voltadas para os cuidados com o recém-nascido, significados expressos pelas puérperas, permitindo assim conhecer os aspectos e fenômenos da vida social de outro modo inacessível. É importante destacar também que, visando garantir o anonimato das participantes, as entrevistas foram codificadas por letras e números, sendo utilizada a letra A seguida de um número conforme as entrevistas eram realizadas, os participantes foram identificados da seguinte de forma A1, A2, ..., A10.

$\mathrm{O}$ estudo respeitou os preceitos éticos para pesquisa envolvendo seres humanos de acordo com a Resolução 466 de 2012 do Conselho Nacional de Saúde. ${ }^{17}$ A pesquisa foi submetida ao Comitê de Ética em Pesquisa em Seres Humanos (CEP) da Universidade do Estado de Minas Gerais, Unidade Divinópolis e aprovada, sob o parecer 26479419.2.0000.5115.

\section{RESULTADOS}

Em relação às características sociodemográficas das puérperas, a idade compreendeu entre 18 e 31 anos, o grau de escolaridade variou de cursando o ensino médio 
$10 \%$ (1), ensino médio completo $40 \%$ (4), ensino médio incompleto $20 \%$ (2), superior incompleto $10 \%$ (1) e superior completo $20 \%$ (2). Em relação ao estado conjugal, $60 \%$ (6) eram solteiras e $40 \%$ (4) eram casadas.

\section{Categoria 1- Início do pré-natal e qualidade das consultas}

Após análise dos relatos foi possível identificar na fala das entrevistadas a idade gestacional de início nas consultas de pré-natal bem como surgiu em meio às entrevistas aspectos sobre a qualidade da assistência das consultas, sendo possível discriminar em duas subcategorias.

\section{Subcategoria 1- Início do pré-natal}

Foi possível observar diante das falas das puérperas que as UBS não conseguem iniciar o pré-natal conforme o recomendado pelo Ministério da Saúde, no qual preconiza iniciar no primeiro trimestre da gestação, até 13 semanas, ${ }^{18}$ assim, foi observado o atraso do início das consultas relatado pelas participantes: [...] "Na décima terceira mais ou menos" [...] (A4); [...] "Semanas eu não sei não, mas eu estava com mais ou menos uns 5 meses" [...] (A5); [...] "14 semanas porque demorei um pouco para descobrir que estava grávida" [...] (A7);

Identificou-se ainda que o número de consultas está na média do recomendado, uma vez que o Ministério da Saúde recomenda que seja realizado no mínimo seis consultas de pré-natal. ${ }^{18}$ Comprovado por meio do registro da caderneta da gestante, na qual foi informado por ela no momento da entrevista: [...]"Anotado eu tenho só sete mas fui em todas as consultas agendadas"[...] (A1); [...] "Sete consultas porque eu comecei depois, eu estava com 3 meses" [...] (A4); "Fui em 10 consultas de pré-natal" [...] (A9);"Foram 7 consultas"[...] (A10).

\section{Subcategoria 2- Qualidade das con- sultas (troca de médicos, unidades de saúde e pouca participação do enfermeiro):}

Nesta subcategoria, destaca-se a mudança do profissional de saúde, o que in- terfere na qualidade da assistência: [...] "Bom eu iniciei no posto do meu bairro e nós começamos com uma médica e um período depois a médica teve que sair e ai eu passei lá para outro posto e consultei com outro médico obstetra, depois voltei para meu posto com novo médico que era clínico geral e ai depois chegou o médico do meu bairro e ai voltei a consultar com ele" [...] (A1); [...] "Então no bairro que eu morava não tinha médico então me mandaram para outro posto e eu comecei com o médico de lá, depois mudei de bairro e fiz o pré-natal no posto perto da minha casa e era um obstetra", (A9).

Evidencia-se, que as consultas de pré-natal devem ocorrer de forma multidisciplinar para que desta forma seja oferecida melhor qualidade de atendimento e informação para a gestante. ${ }^{18}$ Porém, nas consultas de pré-natal relatadas pelas participantes destaca-se o papel predominante do médico mesmo com a recomendação do Ministério da Saúde orientando para cuidados multidisciplinares: [...] "A primeira consulta foi com a enfermeira e as outras com a médica" [...] (A6); [...] "A primeira consulta foi com a enfermeira onde ela já pediu alguns exames e me encaminhou para a médica ginecologista que deu continuidade" [...] (A7); [...] "No bairro que eu morava, quando comecei o pré-natal não tinha consulta com o enfermeiro mais no outro posto, eu tive sim, e me ajudou bastante, me esclareceu algumas coisa sim" [...] (A9); [...] "Foi o médico, o clínico geral” $[\ldots]$ (A10).

Categoria 2- Orientações recebidas durante as consultas do pré-natal, natureza destas informações e dúvidas das puérperas

$\mathrm{Na}$ categoria dois, observou-se como as orientações foram repassadas durante o pré-natal para as participantes, em qual momento foram esclarecidas e por qual profissional da saúde.

\section{Subcategoria 1- Orientações recebi- das}

As participantes relataram que as in- formações repassadas durante o pré-natal não foram detalhadas, eram superficiais e ficavam restritas a gestação: [...]"Não muito detalhado não, muito superficial" [...] (A1); [...] "Assim sem eu perguntar não, mais eu perguntava, porém as respostas eram bem superficiais, a médica me falava que não era hora ainda, ai eu ficava me questionando quando seria a hora certa de tirar minhas dúvidas" [...] (A7);[...] "Nenhuma orientação recebi, apenas orientações sobre a minha gravidez" [...] (A8). "Não, não recebi, eram sobre a gestação mesmo" [...] (A10).

\section{Subcategoria 2 - Natureza das in- formações recebidas}

Diante as informaçóes que as participantes receberam, foi possível observar que se restringiam a amamentação, não abrangendo outros cuidados importantes com o recém-nascido: [...] "Foi sobre a amamentação, a pega certinha, isso o médico foi bem cuidadoso explicou certinho" [...] (A2); [...] "De amamentação apenas" [...] (A4). "Foi gravidez e amamentação" [...] (A10).

\section{Subcategoria 3 - Principais dúvidas que as puérperas tiveram no pré-natal}

As dúvidas das gestantes ajudam os profissionais de saúde envolvidos com as consultas de pré-natal a passar orientações importantes relacionadas aos questionamentos clínicos, medos e preocupações comuns no período gestacional. Observou-se em meio às entrevistas que as dúvidas das participantes emergiam em torno dos cuidados com o RN: [...] "Eu tinha dúvidas com amamentação, umbigo, banho, porque isso tudo assim a gente que é mãe de primeira viajem sempre tem dúvidas em relação a isso" [...] (A1). [...] "Queria perguntar sobre amamentação, sobre o cuidado com o bebê, sobre o sono, sobre a higiene, tudo isso eu gostaria que ela tivesse esclarecido pra mim pra eu já estar com o preparo melhor com meu bebê" [...] (A3).

As dúvidas das gestantes permaneceram nas consultas do pré-natal, não sendo esclarecidas de forma satisfatória. Mui- 
tas vezes eram restritas as orientações do profissional de saúde: [...] "Uai todas necessárias, porque depois que nasce a gente descobre que não sabe nada, quantas horas ele precisa dormir, de quanto em quanto tempo ele precisa mamar, porque quando a gente está no hospital depois que ganha a cabeça fica ruim não lembro de nenhuma orientação que me passaram, e a primeira consulta com o bebê demora muito até lá, ficamos no escuro, e mesmo assim como é no posto não passam muitas informações, só perguntam se está mamando, não querem saber como está a sendo a amamentação que é muito difícil" [...] (A6); [...] "Como eu falei gostaria de saber o máximo possível, porém não sabia o que perguntar, porque não sabia qual seria a minha dificuldade" [...] (A7); [... "Sim, mas eu não perguntava nada não, pois achava que estava certo a forma que estava sendo as consultas" [...] (A8); [...] "Sobre a questão da amamentação, acho que deixou um pouco a desejar, porque como era o meu primeiro, não sabia nada, acho que muita coisa poderia ter sido explicada, também não perguntava porque eu não tinha curiosidade, a única coisa que eu perguntava era sobre a gestação mesmo, eu não perguntei nada do tipo depois da gestação, eu não tive essa curiosidade de perguntar, e também ninguém me informou" [...] (A9).

\section{Categoria 3 - Educação em saúde em grupos de gestantes}

$\mathrm{O}$ processo de construção na educação em saúde para o pré-natal tem como objetivo garantir o desenvolvimento adequado para a gestação, bem como abordar atividades educativas e explicativas. ${ }^{19}$ As ações educativas realizadas durante o pré-natal por meio da equipe multidisciplinar contribuem diretamente para o acolhimento, atendimento integral a saúde, dessa forma ela terá todas as orientações necessárias, além de proporcionar autonomia e empoderamento para a gestante.

\section{Subcategoria 1- Grupos de gestantes}

Os grupos de gestantes têm um papel educativo importante no pré-natal onde possibilita vivências variadas entre as ges- tantes. Durante a análise dos dados, observou-se que as entrevistadas não receberam convite para participar de grupo de gestante e quando recebiam era apenas para palestras: [...] "Grupo não, só uma palestra amamentação exclusiva” [...] (A3); [...] "Sim, uma palestra sobre amamentação" [...] (A7); [...] "Não fui convidada" [...] (A9); [... "Não, não fui convidada não, nenhum grupo de gestante" [...] (A10).

Ressalta-se que durante as entrevistas uma informação importante levantada sobre a educação em saúde no pré-natal foi que as gestantes recebiam convite para a palestra no hospital de referência do município. O convite era realizado por meio de conhecidos (pessoas leigas ou terceiros) e na Unidade Básica de Saúde, nenhum grupo foi relatado. Além disso, os assuntos abordados eram principalmente com foco nas gestantes: [...] "Sim eu participei, mais não fiquei sabendo pelo meu posto não, eu participei de uma palestra na do hospital da minha cidade, lá falou sobre o parto, qual a diferença do parto normal e da cesariana" [...] (A1); [... "Teve grupo, aqui no hospital da cidade, alguém me falou desse grupo, mas eu não fui, eu não participei porque eu estava no meu horário de serviço" [...] (A2); [...] "Eu fiquei sabendo mais não foi pela equipe da unidade, foi por outra pessoa, uma outra gestante, era uma visita na maternidade pra ajudar a preparar as mulheres, foi lá no hospital da minha cidade, mais não fui perdi a data da inscrição" [...] (A3); [...] "Teve uma palestra no hospital da minha cidade, que foi muito boa, porém abordaram temas não relacionados ao neném, falaram mais sobre o parto, e as diferenças, me fez pensar muito sobre o parto e eu escolhi o normal por causa desta palestra, porque antes de conhecer realmente eu queria acesariana, pois achava que era muito ruim o normal pelos relatos que eu tive das minhas amigas, e fui muito feliz no parto, normal” [...] (A8).

\section{DISCUSSÃO}

Mediante os resultados encontrados, foi possível identificar que as principais ações educativas desenvolvidas durante o pré-natal acerca dos cuidados com o recém-nascido, estavam relacionadas à amamentação. A temática é essencial e diz respeito sobre inúmeros benefícios para a nutrição e desenvolvimento do recém-nascido já comprovados na literatura, e a ausência dessa orientação, resulta no desmame precoce o que traz consequências irrecuperáveis para puérpera e para o recém-nascido. ${ }^{20}$

No entanto, ressalta-se que poucos são os estudos que trazem sobre as outras áreas de cuidado ao recém-nascido, como: o banho, cuidado com o coto umbilical, desenvolvimento do RN, vacinação, padrão de sono, dentre outros. ${ }^{21}$ Fato este comprovado nas falas das entrevistadas onde foi possível observar a insuficiência sobre orientações acerca de outros cuidados outros com o recém-nascido.

Além da ausência de orientações sobre os cuidados com o recém-nascido, outro ponto importante encontrado neste estudo foi que algumas participantes começaram o pré-natal tardiamente, o que acarreta em falhas em toda a assistência que deveria ser prestada a gestante. Vacinas ficam atrasadas, exames de rotina e informações importantes não repassadas às gestantes em tempo ideal de gestação. Estudo revela que o início do pré-natal em tempo oportuno é essencial para que ocorra o diagnóstico e intervenção precoce de condições que possam colocar em risco a vida do recém-nascido e gestante. ${ }^{22}$

Atrelado aos cenários da insuficiência das orientações e início tardio do pré-natal o número de consultas realizadas seguiram a média mínima preconizada, sendo realizadas em média seis consultas com as participantes, porém algumas relatam apenas três consultas. No Brasil, a partir de 2012, foi recomendado que fossem realizadas no mínimo seis consultas de pré-natal. ${ }^{20} \mathrm{De}$ acordo com a Leal et $\mathrm{al}^{23} \mathrm{o}$ atraso no início das consultas de pré-natal e a rotatividade entre profissionais de saúde contribuem em menor número de consultas.

A rotatividade de profissionais de saúde principalmente por médicos, da troca das UBS onde seriam realizadas as con- 
sultas e baixa frequência das consultas com enfermeiro instigam dificuldades no sistema de saúde para o cuidado multidisciplinar no pré-natal. ${ }^{24}$ Estudo revela que durante o pré-natal, o enfermeiro desempenha papel complementar as orientações médicas que visam informar sobre as vacinas, tipos de parto, amamentação, cuidados com o recém-nascido sobre saúde, bem-estar, crescimento, segurança e desenvolvimento e as alterações fisiológicas e emocionais no puerpério. ${ }^{25}$

A equipe multidisciplinar precisa observar e ouvir a gestante, buscando entender suas necessidades para melhor elaboração de um plano de cuidados, com benefícios para mãe e o recém-nascido, evitando possíveis complicações. ${ }^{26}$ Além disso, percebe-se um modelo fragmentado, na qual cada profissional da equipe multidisciplinar realiza sua parte do trabalho em saúde, sem integração com os demais profissionais de saúde, o que dificulta a realização uma assistência pautada na integralidade e na qualidade. ${ }^{27}$

As orientações recebidas nas consultas do pré-natal foram sobre a gravidez, amamentação e higiene com o recém-nascido. Esta realidade esta em consonância com a literatura o que refleti no despreparo de puérperas por consequência da falta de orientações recebidas pelos profissionais de saúde durante o pré-natal. ${ }^{20}$ Este poderia ser um momento de educação para o aprendizado relacionadas com o recém-nascido. ${ }^{25}$

Uma forma de melhorar esse cenário é aumentar o acesso das informações sobre as orientações sobre higiene, cuidados com o coto umbilical, posição de conforto, cuidados com o manejo da criança, posição correta para dormir, cuidados durante o banho, produtos que devem ser evitados na pele do RN, importância e necessidade das imunizações, banho se sol, dentre outros, seria o processo educativo para gestantes. ${ }^{4}$

A educação em saúde poderia auxiliar na melhora do conhecimento e autonomia de puérperas para que pudessem desempenhar com segurança todos os cuidados que cercam o recém-nascido. ${ }^{28}$ Uma opção eficaz para contribuir nesse processo são as oficinas educativas com as gestantes. Neste ambiente seria possível trocar experiências, compartilhar discussões que possibilitassem maior acesso à informação. ${ }^{29}$

Nos grupos de gestantes relatados pelas entrevistadas os assuntos relacionavam-se ao parto e como mencionado acima não aconteceram nas unidades de saúde a que eram vinculadas. Os grupos de gestantes são importantes para fortalecer e até mesmo complementar ações educativas durante o pré-natal relacionadas com os cuidados com o recém-nascido. ${ }^{29}$ Dessa forma, a realidade dos serviços de saúde mencionados pelas participantes não responde às necessidades de saúde e as expectativas que as mulheres sentem durante a gestação. ${ }^{30}$

Diante do desenvolvimento do estudo foi possível afirmar que as ações educativas realizadas no pré-natal voltadas para os cuidados com o recém-nascido nas Unidades Básicas de Saúde aconteceram nas consultas e estavam voltadas para a amamentação. Nesse sentido, este estudo permitiu inferir que o sistema de saúde necessita ampliar reflexões essenciais no pré-natal voltadas para os cuidados com recém-nascido, além disso, valorizar as dúvidas das gestantes. Isto porque, muitas vezes, as dúvidas podem despertar durante as consultas, orientações cruciais sobre outros cuidados com o recém-nascido que vão além da amamentação. Principalmente pelo fato de muitas gestantes serem primigestas. É imperativo a capacitação dos profissionais de saúde envolvidos com o pré-natal para que sejam impulsionados a trabalhar com essa temática por meio de um processo educativo a fim de despertar a autonomia e segurança das gestantes ao assumirem os cuidados com seu recém-nascido.

O estudo aponta como limitações o fato das entrevistas terem ocorrido via telefone, justificadas pelo período de isolamento social ocasionado pela pandemia causada pelo novo Coronavírus em março a maio de 2020. As entrevistas ocorreram em horários de disponibilidade das puérperas, ainda que muitas demonstrassem pressa em responder as perguntas por estarem cuidando do recém-nascido. Embora a competência pelo delineamento metodológico do estudo, o desfecho das conclusões restringe as falas das participantes não sendo possível realizar qualquer associação dos resultados por se tratar de pesquisa qualitativa.

\section{CONCLUSÃO}

As ações educativas durante o pré-natal sobre os cuidados com o recém-nascido quando são realizadas acontecem durante as consultas e contemplam na maioria das vezes sobre a amamentação. Este fato aumenta as dificuldades e despreparo das puérperas em relação a outros cuidados com o recém-nascido que se relacionam com a sua segurança, crescimento e desenvolvimento, preconizados pelo Ministério da Saúde.

\section{REFERÊNCIAS}

1. Alves FLC, Castro EM, Souza FKR, Lira MCPS, Rodrigues FLS, Pereira LP. Grupo de gestantes de alto-risco como estratégia de educação em saúde. Rev Gaúcha Enferm. 2019; 40.

2. Livramento DVP, Backes MTS, Damiani PR, Castillo LDR, Backes DS, Simão MAS. Percepções de gestantes acerca do cuida- do pré-natal na atenção primária à saúde. Rev. Gaúcha Enferm. 2019; 40.

3. Cardoso MD, Ribeiro CMS, Oliveira IB, Andrade PMC, Santos TMB. Percepção de gestantes sobre a organização do serviço/ assistência em um pré-natal de baixo risco de Recife. Revista 


\section{REFERÊNCIAS}

de Pesquisa: Cuidado é Fundamental Online. 2016; 8(4):50175024.

4. Dias EG, Novaes CCM, Santos IR, Silva SX, Alves JCS. Conhecimento de gestantes de uma unidade de saúde sobre os cuidados com o recém-nascido. Rev. Inova Saúde. 2019; 9(1).

5. Fagundes DQ, Oliveira AE. Educação em saúde no pré-natal a partir do referencial teórico de Paulo Freire. Trab. educ. saúde [online]. 2017; 15(1):223-43.

6. Andrade RD, Santos JS, Maia MAC, Mello DF. Fatores relacionados à saúde da mulher no puerpério e repercussões na saúde da criança. Esc. Anna Nery. 2015; 19(1):181-186.

7. Souza NA, Queiroz LLC, Queiroz RCCS, Ribeiro RSF, Fonseca MDSS. Perfil epidemiológico das gestantes atendidas na consulta de pré-natal de uma unidade básica de saúde. Rev. Ciênc. Saúde. 2013; 15(1):28-38.

8. Viellas EF, Domingues RMSM, Dias MAB, Gama SGNID, Theme Filha MM, Costa JVD, et al. Assistência pré-natal no Brasil. Cad. Saúde Pública. 2014; 30:S85-S100.

9. Tomasi E, Fernandes PAA, Fischer T, Siqueira FCV, Silveira DSD, Duro SMS, et al. Qualidade da atenção pré-natal na rede básica de saúde do Brasil: indicadores e desigualdades sociais. Cad. Saúde Pública, Rio de Janeiro. 2017; 33(3).

10. Silva EP, Lima RT, Osorio MM. Impacto de estratégias educacionais no pré-natal de baixo risco: revisão sistemática de ensaios clínicos randomizados. Ciênc. saúde coletiva. 2016; 21(9):2935-2948.

11. Matos MR, Condas BA, Cappelletti C, Skupien SV. Atuação do profissional enfermeiro no pré-natal: educando para a saúde. Educere. 2016.

12. Bosi MLM. Pesquisa qualitativa em saúde coletiva: panorama e desafios. Ciência \& Saúde Coletiva. 2012; 17:575-586

13. Instituto Brasileiro de Geografia e Estatística (IBGE). Cidades e Estados: Município Divinópolis - Minas Gerais; 2020. Disponivel em:< https://www.ibge.gov.br/cidades-e-estados/mg/ divinopolis.html>.

14. Ministério da Saúde (BR). Portaria n 2.436, de 21 de setembro de 2017. Aprova a Política Nacional de Atenção Básica, estabelecendo a revisão de diretrizes para a organização da Atenção Básica, no âmbito do Sistema Único de Saúde (SUS). Diário Oficial União. 22 set 2017; Seção 1.

15. Nascimento LCN, Souza TV, Oliveira ICS, Moraes JRMM, Aguiar RCB, Silva LF. Theoretic al saturation in qualitative research: na experience report in interview with school children. Revista Brasileira de Enfermagem. 2018; 71(1):228-233.

16. Bardin L. Análise de conteúdo. São Paulo: Edições 70; 2011.

17. Ministério da Saúde (BR). Resolução n 466, de 12 de dezembro de 2012. Resolução n 466, de 12 de dezembro de 2012. Diretrizes e normas regulamentadoras de pesquisas envolvendo seres humanos. Diário Oficial União. 12 dez 2012.

18. BRASIL. Caderno de Atenção Básica. Atenção ao Pré-Natal de Baixo Risco [internet]. Brasil: Governo Federal; 2012 [acesso em 21 set 2019]. Disponivel em:http://bvsms.saude.gov.br/bvs/ publicacoes/cadernos_atencao_basica_32_prenatal.pdf.

19. Camillo BS; Nietsche EA, Salbego C, Cassenote LG, Osto DSD, Bäck $A$. Ações de educação em saúde na atenção primária a gestantes e puérperas: revisão integrativa. Rev. enferm. UFPE online. 2016; 4894-4901.

20. Rocha FNPS, Patrício FDB, Passos MNSD, Lima SWOD, Nunes MGS. Caracterização do conhecimento das puérperas acerca do aleitamento materno. Rev. enferm. UFPE online. 2018; 2386-2392.

21. Carvalho MS, Aragão MD, Oliveira SJGS. Educação em saúde durante o pré-natal com foco nos cuidados relacionados ao recém-nascido. Caderno de Graduação-Ciências Biológicas e da Saúde-UNIT-SERGIPE. 2016; 3(3):157.

22. Sehnem GD, Saldanha LS, Arboit J, Ribeiro AC, Paula FM. Consulta de pré-natal na atenção primária à saúde: fragilidades e potencialidades da intervenção de enfermeiros brasileiros. Revista de Enfermagem Referência. 2019; 5(1).

23. Leal MC, Pereira APE, Viellas EF, Domingues RMSM, Gama SGN. Assistência pré-natal na rede pública do Brasil. Revista de Saúde Pública. 2020; 54(08).

24. Laporte ASCM, Zangirolani LTO, Medeiros MAT. Atenção nutricional ao pré-natal e puerpério, na perspectiva da integralidade, em um município da Baixada Santista, São Paulo, Brasil. Revista Brasileira de Saúde Materno Infantil. 2020; 20(1):145155.

25. Oliveira JCS, Fermino BPD, Conceição EPM, Navarro JP. Assistência pré-natal realizada por enfermeiros: o olhar da puérpera. Revista de Enfermagem do Centro-Oeste Mineiro. 2015; 5(2).

26. Castro LS, Rached CDA. Acolhimento humanizado no cuidado pré natal as gestantes da ESF. International Journal of Health Management Review. 2019; 5(3).

27. Nogueira CMCS, Sousa CNS, Nóbrega LLR, Sales LKO, Morais FRR. Assistência ao pré-natal e as práticas desenvolvidas pela equipe de saúde: revisão integrativa. Revista de Pesquisa: Cuidado é Fundamental Online. 2017; 9(1):279-288.

28. Lima VKS, Hollanda GSE, Oliveira BMM, Oliveira IG, Santos LVF, Carvalo CML. Educação em saúde para gestantes: a busca pelo empoderamento materno no ciclo gravídico-puerperal. Revista de Pesquisa: Cuidado é Fundamental. 2019; 11(4):968975.

29. Pinto JSR, Silveira V, Araújo C, Ferreira F, Firmes M. Oficina de sensibilização para gestantes: construindo práticas para o autocuidado da mulher e do recém-nascido. Revista Ciência em Extensão. 2019; 15(3): 18-29.

30. Cotrim TS, Rodrigues VS, Torres MRF, Rodrigues VFF, Guimarães NB. Avaliação do pré-natal de usuárias cadastradas em uma Estratégia Saúde da Família de uma cidade do Pará. Revista Artigos.com. 2020; 17:3466. 